\title{
Experiencias de estudiantes mapuches en formación inicial docente en contexto de diversidad
}

\author{
Eliana Marcela Ortiz Velosa \\ Universidad Católica de Temuco - Temuco - IX región - Chile \\ Enrique Riquelme Mella \\ Universidad Católica de Temuco - Temuco - IX región - Chile
}

\begin{abstract}
Resumen
La inserción y permanencia de los estudiantes de culturas minoritarias involuntarias en el sistema educativo implica una serie de tensiones académicas y socio-efectivas que pueden influir en su desempeño académico y en la forma en la que se relacionan con el sistema educativo. El objetivo de este ensayo es presentar una reflexión sobre algunas de las problemáticas que los estudiantes indígenas experimentan en la universidad, en tanto reproductora de los ideales monoculturales del sistema de educación. Para ello reflexionaremos sobre la base teórica de la educación desde una perspectiva intercultural en relación al rol de las universidades y de la formación inicial de profesores. Las proyecciones del ensayo se refieren a las implicancias epistemológicas y praxiológicas en tanto aporte a la base de conocimientos acerca de la educación superior en contextos de diversidad social y cultural para una educación que dé cuenta de las variaciones culturales en la formación de profesores.
\end{abstract}

Palabras clave: Estudiantes universitarios; diversidad cultural, grupos minoritarios.

\section{The experiences of the mapuche students at the start of their teaching training under a diverse context}

\begin{abstract}
A series of academic and socio-effective strains arise with the insertion and permanence of involuntary minority culture students in the education system which can influence their academic performance as also in the way that they relate to the education system. The purpose of this essay is to give some forethought regarding the problems indigenous students face in the university, where the education system only present monocultural ideals. For this we will contemplate the theoretical basis of education from an intercultural perspective in regards to the participation of the universities and the initial formation of the professors. The outreach of the essay is in reference to the epistemological and praxeological implications that contribute to a knowledge based on a superior education with a social and cultural diversity context for an education where the professors have been formed with cultural variations.
\end{abstract}

Keywords: College Students; cultural diversity; minority groups.

\section{Experiências de estudantes mapuches em formação inicial docente no contexto de diversidade}

\begin{abstract}
Resumo
A inserção e a permanência dos estudantes de culturas minoritárias involuntárias no sistema educativo implicam uma série de tensões acadêmicas e socioafetivas que podem influenciar em seu desempenho acadêmico e na forma como se relacionam com o sistema educativo. $\mathrm{O}$ objetivo deste ensaio é apresentar uma reflexão sobre algumas das problemáticas que os estudantes indígenas experimentam na universidade, em tanto reprodutora dos ideais de monoculturais do sistema de educação. Para isso refletimos sobre a base teórica da educação a partir uma perspectiva intercultural em relação ao rol das universidades e da formação inicial de professores. As projeções do ensaio referem-se às implicações epistemológicas e praxiológicas em tanto aporte à base de conhecimentos sobre a educação superior nos contextos de diversidade social e cultural para uma educação que dê conta das variações culturais na formação de professores.
\end{abstract}

Palavras-chave: Estudantes Universitários; diversidade cultural, grupos minoritários. 


\section{Introducción}

La problemática que se aborda en el ensayo está relacionada con las experiencias universitarias de estudiantes mapuches en su proceso de formación inicial docente en la región de La Araucanía al sur de Chile. Existen diversos factores que se han conjugado para hacer de la experiencia universitaria un escenario más complejo y tensionante para los estudiantes pertenecientes a grupos originarios (Brunner, 2015). Dichas tensiones pueden surgir, desde la perspectiva del estudiante, por factores académicos relacionados con el desarrollo de competencias básicas previas al ingreso a la universidad (Hall, 2012), por el tipo de apoyo académico que pueda ofrecer la institución (Milne, Creedy, \& West, 2016), pero también con dimensiones socio-afectivas relacionadas con discriminación y racismo Institucional, de los profesores o de sus pares que no pertenecen a grupos originarios (Oliver \& cols., 2013)

Por otro lado, desde la Universidad como institución educativa, las tensiones surgen por aspectos administrativos, cambios legales y proyecciones curriculares que responden a las exigencias del contexto nacional y que no siempre logran responder a las necesidades del contexto local respecto a diversidad social y cultural (Wit, 2011). En efecto, las instituciones universitarias reconocen la diversidad social y cultural de sus estudiantes desde una perspectiva funcional, estadística y política, generando rutas de acceso a la Educación Superior y posibilidades de acceso a estudiantes que tradicionalmente no ingresaban a la universidad, como es el caso de los estudiantes mapuches (Arancibia, Guerrero, Hernández, Maldonado, \& Román, 2014). Sin embargo, aunque se releva la riqueza de la diversidad cultural en los estudiantes universitarios, y se toman iniciativas de acompañamiento, las universidades no han optado por una postura formal respecto de la formación de profesionales pertenecientes a las culturas minoritarias voluntarias e involuntarias, tampoco se implementan acciones integrales para solucionar los problemas de racismo, discriminación propios de la educación monocultural del sistema educativo chileno.

En este sentido, la educación que se ofrece en la Universidad continúa con la perspectiva monocultural del sistema educativo, lo que - en un contexto de diversidad social y cultural - también genera tensiones. La función de este tipo de educación es capacitar individuos para el mundo del trabajo e impartir ideologías que se supone son deseables para la sociedad moderna, invisibilizando las necesidades propias del contexto caracterizado por la diversidad social y cultural (Atta-Alla, 2012; Olivera, 2013). La reproducción de la monoculturalidad en los espacios de educación, reproduce conductas discriminatorias excluyentes y racistas, explicitas o implícitas, entre las relaciones de los diferentes actores educativos (Carignan, Sanders, \& Pordavood, 2005; Mampaey \& Zanoni, 2015; Veldman \& Guilfoyle, 2013)

Dichas tensiones podrían eventualmente conducir a los estudiantes a un fracaso académico (Milne, Creedy, \& West, 2016) frustración de los profesores y de los estudiantes (Bishop, Berryman, Wearmouth, Peter, \& Clapham, 2012), conflictos personales, académicos y sociales (Watson
\& cols., 2002), crisis en su identidad cultural (Cook-Sather, 2015) y una baja tasa de retención de estudiantes pertenecientes a los grupos minoritarios (Chirgwin, 2015).

En resumen, se presenta un escenario en el que se mantiene una lógica mono-cultural en la educación universitaria en la medida que se implementan estrategias de acompañamiento y apoyos a estudiantes de culturas minoritarias, sin considerar las variaciones culturales. En efecto, en Chile hay una exploración casi nula de las experiencias universitarias de los estudiantes mapuches y sus profesores universitarios narradas por los propios actores, se hace urgente explorar este problema desde las experiencias universitarias relatadas desde la propia voz de los estudiantes en las dimensiones académicas y socio-afectivas, las cuales podrían aportar a los procesos de inserción, acompañamiento, permanencia y formación de los estudiantes de pueblos originarios en calidad de futuros profesores.

Relevar la voz de los profesores y estudiantes que comparten experiencias universitarias en contextos de diversidad social y cultural generará conocimientos que permitirán contribuir a la formación inicial de profesores pertinente para distintos contextos, desde la perspectiva de la interculturalidad crítica que atiende la diversidad.

\section{Diversidad y Educación}

En Chile, de acuerdo a la Ley General de Educación 20.370 publicada en 2009 , la educación se define como el proceso de aprendizaje a lo largo de vida y tiene como fin la formación permanente, universal y de calidad, propendiendo a la equidad al brindarles a todos los estudiantes las mismas oportunidades. Así mismo, la educación "se enmarca en el respeto y valoración de los derechos humanos y de las libertades fundamentales, de la diversidad multicultural y de la paz..." (Artículo 2). Se añade en el artículo 3, que la educación es un derecho constitucional que se inspira, entre otros, en los principios de la universalidad, calidad, equidad, diversidad, flexibilidad, integración e interculturalidad. Es decir, que el sistema de educación chileno debe garantizar educación para todos los niños y jóvenes del país, buscando las mismas oportunidades de calidad, respetando la diversidad cultural, religiosa y social. En este sentido, el sistema debe reconocer al individuo en su identidad cultural, facilitando las adecuaciones curriculares que permitan considerar la lengua, cosmovisión y facilite el diálogo armónico entre las personas de la sociedad mayoritaria y la cultura, lengua, cosmovisión e historia de las personas de pueblo originarios minoritarios (Ministerio de Educación Nacional, 2009).

El contexto social y cultural chileno es diverso, por la presencia significativa de pueblos indígenas como los Aymara, Rapa Nui, Quechua, Atacameño, Coya, Kewesqar, Yagán, Diaguita y Mapuche que ascienden al 9,1\% de un total de 16.634.603 habitantes (Encuesta de caracterización Socioeconómica Nacional [CASEN], 2013). Del total de las personas que se han identificado como pertenecientes a pueblos originarios, el $84,4 \%$ se identifican como mapuche 
(CASEN, 2013), siendo entonces el grupo más representativo entre las culturas minoritarias de Chile.

Adicionalmente al número habitantes pertenecientes a pueblos originarios se suman inmigrantes que ingresan al país desde diferentes partes del mundo. El Ministerio de Relaciones Exteriores reportó un incremento de inmigrantes del $50 \%$ entre los años 2004 a 2010, para el año 2014 se estimó que el $2,3 \%$ de la población en Chile son inmigrantes, es decir 411 mil personas. De esta cifra se estima que el 56,8\% de ellos provienen de países sudamericanos (Departamento de Extranjería y Migración Ministerio del interior y Seguridad Pública, 2016). Por ejemplo, los inmigrantes peruanos representan el $31,7 \%$, siendo la comunidad migrante con mayor magnitud, seguida por la comunidad argentina con el $16,3 \%$.

La diversidad social y cultural nacional se evidencia en la coexistencia de diferentes sociedades; la chilena definida como el grupo mayoritario y dominante, la indígena, cultura minoritaria involuntaria, y por lo tanto, sociedad colonizada por la cultura dominante y los migrantes, también pertenecientes a los grupos minoritarios pero voluntaria, los cuales están en constante movilidad (Ogbu \& Simons, 1998)

Este escenario, en el marco legal descrito previamente, desafía al sistema educativo nacional en distintas dimensiones: a) en el ámbito de las políticas públicas porque se requieren normativas y planes de implementación para garantizar el cumplimiento de las leyes que promueven la educación de calidad para todos, de formas que respondan a las necesidades propias de los contextos de diversidad social y cultural (Hevia, 2010), b) presenta un deber desde el punto de vista de la gestión educativa, porque las escuelas y universidades deben garantizar el acceso, la integración y acompañamiento de todos sus estudiantes sin discriminar ningún nivel social o identidad cultural (Merin, 2013) y, c) en lo pedagógico, porque las instituciones de Educación Superior deben descontinuar la impronta monocultural heredada por el eurocentrismo, rescatando las epistemologías latinoamericanas para integrarlas al currículo y a los procesos de formación.

De esta forma, aunque existe un reconocimiento legal y demográfico de la diversidad social y cultural, Chile históricamente ha mantenido desigualdad social y educativa (Diez, 2013). La desigualdad -desde el punto de vista social- se manifiesta en la pobreza, falta de cobertura de las necesidades básicas, abandono por parte del Estado e injusticias asociadas al despojo territorial y prácticas discriminatorias hacia las minorías sociales o étnicas comúnmente relacionadas con la vulnerabilidad. En el sistema de educación, la desigualdad se ha manifestado en la inequidad de oportunidades y calidad educativa, la falta de cobertura y la reproducción de una educación monocultural reproductora de racismo y discriminación (Fernandes \& Ferrão, 2013; Quilaqueo, Quintriqueo, \& Torres, 2014).

En efecto, la educación monocultural ha sido impuesta desde la colonia, y proviene de la tradición occidental (Brunner, 2015). Por años la escuela y las universidades han perpetuado este paradigma negándose a validar e incluir los saberes no occidentales, como los saberes indígenas, para mejorar las oportunidades de aprendizaje de los estudiantes que pertenecen a culturas minoritarias y dominadas (Mampaey \& Zanoni, 2015).

De esta forma, la educación monocultural puede traducirse en racismo institucional, en tanto la institución educativa no logra ofrecer su formación de una forma adecuada para las personas de culturas minoritarias (Lea, 2000; Meersohn, 2005) el cual se manifiesta a través de actos no evidentes, ni explícitamente agresivos de discriminación, por lo tanto las consecuencias no son detectadas con facilidad por los diferentes actores educativos pero sus efectos son negativos en los procesos educativos (Carignan \& cols., 2005; Mampaey \& Zanoni, 2015; Veldman \& Guilfoyle, 2013).

Este tipo de educación monocultural se mantiene y reproduce a través de diferentes procesos: a) fundamentando la noción de la realidad desde la objetivación, b) invisibilizando las representaciones humanas y lingüísticas y c) separando el conocimiento de las creencias y cultura de los sujetos (Quintriqueo, Quilaqueo, Peña, \& Muñoz, 2015); además, d) reproduciendo las relaciones y prácticas coloniales a través de la dominación epistémica eurocentrista (Kerr, 2014), e) desde un plano afectivo este tipo de educación se presenta en la negación de las formas culturalmente propias de regular la emoción de los niños, en la búsqueda de una homogeneidad que refleje los lineamientos occidentales (Mella, Rapiman, Millan, \& Antileo, 2016) y, d) en las prácticas educativas, la monoculturalidad se expresa a través del monolingüismo, la exclusión de las religiones no occidentales y por los contenidos curriculares no occidentales (Mampaey \& Zanoni, 2015).

Desde esta racionalidad la presencia del "otro" y la diversidad social y cultural puede relacionarse con un problema que puede ser tratado con la educación como instrumento para homogeneizar y sistematizar cualquier razonamiento cultural diferente (López, 2012). O bien, puede abordarse mediante programas de integración dirigidos a estudiantes que tradicionalmente han sido excluidos (Williamson \& Navarrete, 2014). Como hemos señalado, el resultado sigue siendo la mantención de una forma única de abordar la diversidad que mantiene la objetividad como argumento para obligar, y el racismo y la discriminación como resultado de la negación de las diferencias epistémicas y la imposición de una racionalidad dominante.

Este es un estado transversal en la educación obligatoria Chilena. Es decir, estudiantes de culturas minoritarias involuntarias deben formar parte de esta educación monocultural desde la educación preescolar hasta la educación básica y media. La Formación Técnica y Universitaria es también parte de esta formación monocultural (Quilaqueo \& cols., 2014).

\section{Formación inicial de profesores}

En la Educación Superior chilena la cobertura y acceso ha aumentado en los últimos años, ascendiendo a un $54,9 \%$ de cobertura de población estudiantil entre 18 y 
24 años (Navarrete, Candia, \& Puchi, 2013). El Sistema de Información de Educación Superior (SIES) en el año 2011 reportó 117 instituciones de Educación Superior, 59 universidades y una matrícula que llegó a cerca de un millón de estudiantes; en el año 2012 estos datos superaron el millón cien mil estudiantes.

Para el año 2015, según el Consejo Nacional de Educación (CNED) el total de estudiantes en Educación Superior a nivel nacional fue de 1.152.125, distribuidos en 262 carreras. De este total, $78.349(6,3 \%)$ son estudiantes universitarios en Pedagogía; en la Región de la Araucanía 8 universidades ofrecen programas de Pedagogía en 10 áreas disciplinarias, el total de estudiantes en la región es de 4.270 $(0,4 \%)$ (mifuturo.cl). Lamentablemente, las estadísticas del CNED y del Ministerio de Educación (MINEDUC), no permite un análisis según ascendencia de los estudiantes.

A pesar de no contar con datos del MINEDUC, diferentes universidades de la Araucanía han reportado un incremento significativo de estudiantes indígenas matriculados en programas de pregrado. De acuerdo a Blanco y Meneses (2011) en el 2009 la Universidad de la Frontera incrementó el número de estudiantes en un 18,6\%; En el año 2016, el $21 \%$ de estudiantes de la Universidad Autónoma se identifican como pertenecientes a pueblos originarios. Finalmente, el $27,61 \%$ de los estudiantes de la Universidad Católica de Temuco (UCT) se identifican como pertenecientes a pueblos originarios, de este porcentaje el $28,67 \%$ pertenecen a la Facultad de Educación (Sistema de Información Institucional KIMN, UC Temuco).

Si bien la Educación Superior en Chile dejó de ser un privilegio de un grupo social de élite para masificarse y generar rutas de acceso para los estudiantes mapuches (Brunner, 2015) y las políticas educativas han explicitado objetivos de equidad y calidad en la oferta educativa de las universidades regionales, aún existen brechas sociales que han mantenido la inequidad (Cifuentes, 2015). Por ejemplo, en la región de La Araucanía el ingreso de estudiantes mapuches a la universidad sigue siendo bajo respecto de los estudiantes no mapuches; para el año 2005 el ingreso fue del 7\%, es decir de 39.142 estudiantes; adicionalmente, las condiciones de ingreso universitario están relacionadas con experiencias de vulnerabilidad social y discriminación (Navarrete \& cols., 2013). En este sentido, el aumento de la cobertura en la Educación Superior no ha significado una disminución de la desigualdad, ni del racismo, tampoco ha implicado mejorar la calidad en los procesos de formación profesional.

En particular, las carreras de las facultades de educación ofrecen prácticas pedagógicas tradicionales y monoculturales (Turra, Ferrada, \& Villena, 2013; Turra, Valdebenito, \& Torres, 2015), es por ello que igualmente se han convertido en actividades discriminatorias, dado que 1) la universidad como institución ha pretendido históricamente la homogenización aspirando a que todos los estudiantes se formen en la misma escala valórica (Cornejo, 2012); 2) Un profesor formado en un paradigma de homogeneidad tiende a replicarla, por ello la diversidad en el aula es vista como un problema (Apablaza, 2014; López, 2012; Riquelme, Daniel,
Segundo, \& Elisa, 2016); 3) La discriminación por parte de profesores hacia los estudiantes indígenas puede ser explícita o implícita, por ejemplo, desde la etiquetación hasta la creación de prejuicios (Carignan \& cols., 2005; Schmelkes, 2010) y 4) las prácticas discriminatorias se evidencian en el currículo a través de la exclusión de los saberes indígenas y de la reproducción de estereotipos desde los contenidos occidentales.

La Formación Inicial de Profesores (FIP) es entonces clave en la dinámica de reproducción de una educación monocultural y racista que excluye a quienes no forman parte de esta cultura dominante, estandarizando a los estudiantes a través de las prácticas pedagógicas y evaluativas (Perrenoud, 2007); En efecto, la formación inicial docente no está respondiendo a las necesidades educativas para un contexto de diversidad social y cultural, en particular para la formación de profesores en contexto mapuche (Cupples \& Glynn, 2014; Turra \& cols., 2015).

Lo anterior es aún más complejo para estudiantes de culturas minoritarias, puesto que, durante la FIP, pueden vivir experiencias universitarias tensionadas asociadas a discriminación y racismo, lo que es estable en la educación nacional, pero además es una formación en donde debe prepararse para formar desde un modelo estandarizado, pese a las necesidades de los diferentes contextos, como el indígena (Turra \& cols., 2013).

Es decir que los estudiantes de pedagogía no sólo enfrentan tensiones en su formación profesional sino que, al ejercer como profesores deben enfrentar distintas formas culturales lo que requeriría especificidades curriculares y pedagógicas para lo cual no fueron formados (Quintriqueo, 2010). Las tensiones en el proceso de formación inicial pueden surgir en el ámbito académico: a) en relación a las propias características del estudiante, por ejemplo en sus motivaciones de ingreso a la universidad, las aspiraciones como futuro profesional, en el desarrollo de sus competencias disciplinares y en las necesidades académicas (West, Usher, Foster, \& Stewart, 2014) b) en relación a la institución universitaria respecto del proceso de ingreso, el apoyo académico para el estudiante y la claridad de los canales de comunicación (Watson \& cols., 2002) c) en relación a las oportunidades de aprendizaje, es decir la posibilidad de relacionar sus conocimientos previos a los nuevos conocimientos disciplinares, el respeto hacia sus conocimientos y los ambientes para el aprendizaje (Hall, 2012), y d) finalmente, las redes y relaciones relacionadas al apoyo académico de sus profesores y pares (Milne \& cols., 2016).

De igual manera, las tensiones en las experiencias universitarias pueden surgir en el ámbito socio-afectivo: a) en relación a las propias características del estudiante, por ejemplo su identidad, origen étnico y la capacidad de desarrollar una doble racionalidad indígena-occidental (Quilaqueo, 2012; Shoyer, Leshem, \& Boylan, 2016), b) en relación a la institución universitaria respecto al respecto cultural percibido por el estudiante, la posibilidad de integrar su cultura a la vida universitaria y las declaraciones instituciones respecto de la diversidad social y cultural (Oliver \& cols., 2013; Wat- 
son \& cols., 2002) c) finalmente, las redes y relaciones con sus profesores, pares indígenas, pares no indígenas, apoyo de su familia y percepción del respeto y aceptación (Milne \& cols., 2016).

En resumen, desde la visión de los estudiantes, las experiencias universitarias en contextos de diversidad social y cultural se complejizan en lo académico y socio-afectivo debido al carácter mono-cultural de la oferta de la educación superior.

Lo anteriormente señalado se complementa desde la perspectiva del profesorado universitario sobre la experiencia universitaria de estudiantes de culturas minoritarias, quienes no pueden enseñar aquello que no poseen y mantienen prácticas discriminatorias y racistas (Riquelme \& cols., 2016).

Díaz-Aguado (2004) afirma que los profesores provenientes de la cultura dominante logran reconocer la diversidad social y cultural en su discurso, pero en las prácticas pedagógicas, en el contexto de la escuela, no saben cómo establecer puentes que les permita contribuir a un aprendizaje relevante para los estudiantes, especialmente para los de cultura minoritaria. Aquello conduce a los profesores a la búsqueda errada de la 'receta' que les permita mejorar su práctica pedagógica sin cuestionarse los problemas a la base de la educación en contextos diversos los cuales hemos mencionado.

Lo anterior se asocia a frustraciones en sus prácticas pedagógicas debido a las pocas herramientas para atender la diversidad y por el bajo desarrollo de las competencias interculturales (Díaz-Aguado, 2004; Milne \& cols., 2016) y para la institución implica el incumplimiento de los criterios de calidad relacionados con la retención de estudiantes y tasas de aprobación (Díaz \& Careaga, 2010).

En el caso específico de los profesores universitarios, se ha evidenciado la importancia de la formación permanente que no solo contribuya al desarrollo de competencias específicas para la innovación del quehacer pedagógico sino que además, forme competencias valóricas (Gómez \& Navío, 2013).

El desarrollo de estas competencias permite al profesor adquirir habilidades para el análisis de los diferentes contextos socioculturales, la exploración de metodologías para la enseñanza en contextos diversos, prácticas pedagógicas reflexivas y el mejoramiento a partir de la autoevaluación.

Un ejemplo de lo anterior se evidencia en el estudio de Niculescu y Percec (2015) donde profesores de diferentes países compartieron sus experiencias y metodologías de la práctica pedagógica en contextos de diversidad cultural. El estudio devela que los profesores universitarios requieren adoptar una actitud de aceptación respecto de la diversidad lo cual se evidencia en el respeto de los sujetos de diferentes culturas, en la práctica de escuchar activamente a los estudiantes. Adicionalmente los profesores reconocen la necesidad de despojarse de la discriminación y el prejuicio para poder desarrollar competencias interculturales y lingüísticas que permitan mejorar la calidad de vida, el aprendizaje y la adaptación de los estudiantes provenientes de culturas minoritarias (Niculescu \& Percec, 2015).

\section{Algunos puntos para concluir}

Desde las perspectivas de estudiantes universitarios, diversos estudios destacan la voz de los estudiantes para develar las tensiones, demandas, obstáculos y oportunidades que surgen en el contexto de diversidad social y cultural en las universidades. Por ejemplo estudios con universitarios Maorí se han enfocado en investigar las experiencias universitarias para: a) develar cuáles son los desafíos de los estudiantes indígenas en la universidad (Chirgwin, 2015) b) develar cuáles factores inciden en la permanencia, éxito o fracaso (Hall, Rata, \& Adds, 2013), c) conocer la perspectiva y experiencia respecto de la educación en contextos de diversidad social, cultural y étnica, narrada por los profesores (Cook-Sather, 2015) y por los estudiantes (Lewthwaite, Osborne, \& Boon, 2015) finalmente, d) indagar por las experiencias de práctica pedagógica con el objetivo de buscar nuevas y mejores alternativas para la formación inicial de profesores (Bishop \& cols., 2012).

En la misma línea investigativa otros estudios a nivel mundial resaltan las experiencias de los estudiantes universitarios, por ejemplo en Finlandia se ha indagado sobre las posibilidades de diálogo intercultural en la universidad, generando con ello nuevas perspectivas en los estudiantes y posibilidades de experiencias con diferentes culturas (Lehtomäki, Moate, \& Posti-Ahokas, 2015). En Egipto se analizó el discurso de los estudiantes y profesores de diferentes culturas para develar actos de discriminación y prejuicios que acontecen en el aula (Atta-Alla, 2012), y en África se investigó acerca de los procesos de enseñanza en relación a la pertinencia cultural (Die, 2011).

En América Latina, la Universidad Autónoma de Chapingo en México demostró que los estudiantes indígenas, de diferentes cursos y carreras universitarias, experimentan problemas académicos relacionados con el bajo rendimiento académico, inseguridad, reprobación y deserción. De igual manera experimentan problemas no académicos como discriminación, soledad, falta de redes de apoyo, problemas con sus pares, mala comunicación y problemas adaptación (Chávez, 2008).

Desde diferentes puntos de vista, dichos estudios buscan aportar al conocimiento y explorar soluciones a las problemáticas que surgen en los espacios educativos asociados a la diversidad social y cultural, los cuales pueden estar relacionados con aspectos académicos y no académicos. Los estudiantes pertenecientes a culturas minoritarias reconocen la presencia de algunos conflictos entre con sus profesores, personal administrativo y estudiantes de la cultura mayoritaria, que surgen entre otros aspectos, por fallas en la comunicación, el ambiente en el que se produce el aprendizaje y actitudes relacionadas con el racismo y la discriminación. Como posibles soluciones, los mismos estudiantes proponen que las relaciones se enriquezcan con el diálogo respetuoso, se reconozcan en incluyan los diferentes saberes culturales y se construyan relaciones interculturales (Expósito, Olmedo, Pegalajar, \& Tomé, 2014). 


\section{Proyecciones}

Tanto profesores como estudiantes de culturas minoritarias relevan la importancia de atender la diversidad social y cultural en la Educación Superior para que la educación sea pertinente a las necesidades del contexto y le permitan al estudiante de la cultura minoritaria, en este caso indígena, mantener su identidad, su cultura y le permita relacionar con otros actores educativos con base en la educación con un enfoque intercultural que surja en un contexto de relaciones basadas en la igualdad y el diálogo intercultural.

Especificamente en Chile, las relaciones interculturales entre la cultura dominante occidental chilena y la cultura minoritaria indígena han estado basadas en la injusticia social, la discriminación y el racismo heredado por la época de la colonia y que se ha reproducido a través de los sistemas de educación. En la Educación Superior se presentan realidades de inequidad evidenciadas en las desigualdades de las experiencias académicas previas a la educación universitaria lo que dificulta el ingreso y la permanencia en los programas de formación profesional en especial en las universidades regionales, en las que se reportan mayor cantidad de estudiantes de origen étnico y de zonas rurales.

En la Región de La Araucanía la formación inicial de profesores presenta una matrícula considerable de estudiantes indígenas mapuches, lo que implica un desafío para la atención de la diversidad social y cultural en los proceso de enseñanza y aprendizaje. Considerando que los profesores tienen una incidencia directa con la formación de niños y niñas del país en la escuela y con ello podrán descontinuar con el patrón de la monocultural presente en la educación chilena, es fundamental construir conocimiento de base que aporte a los procesos de formación inicial de profesores pertinente para las necesidades propias de la diversidad social y cultural de la Región.

En este contexto se hace necesario no solo explorar las experiencias de los estudiantes universitarios de origen mapuche de programas de pedagogía en la región de La Araucanía, sino también las experiencias de los profesores de pedagogía pertenecientes a la cultura dominante para develar la voz de los actores educativos respecto de los aspectos académicos y socio-afectivos que favorecen u obstaculizan el proceso de formación inicial de profesores que se desempeñarán en contextos de diversidad cultural.

Efectivamente, creemos que no es posible aportar al cambio educativo sin la construcción de un conocimiento que surja de quienes forman parte de la dinámica educativa, en particular en la universidad. Reconocer la voz de los estudiantes universitarios pertenecientes a los grupos minoritarios puede generar conocimiento que fundamente la implementación de una formación universitaria desde la perspectiva intercultural crítica en la que se reconozca no sólo la existencia de la diversidad social y cultural sino que también se proyecte disminuir los efectos de la educación monocultural. Así mismo, la voz de los estudiantes puede develar las estrategias que desarrollan los estudiantes de las minorías para lograr el éxito académico a pesar de las tensiones vivenciadas en su experiencia universitaria.

\section{Referencias}

Apablaza, M. (2014). Representaciones sociales de profesores respecto de la diversidad escolar en relación a los contextos de desempeño profesional, prácticas y formación inicial. Estudios pedagógicos, $X L(1), 7-24$.

Arancibia, M., Guerrero, D., Hernández, V., Maldonado, M., \& Román, D. (2014). Análisis de los significados de estudiantes universitarios indígenas en torno a su proceso de inclusión a la educación superior. Psicoperctectivas Inividuo y sociedad, 13(1), 35-45.

Atta-Alla, M. (2012). Egypt Education System: A Monocultural Education in a Multicultural Society. Journal of Sociological Research, 3(2), 476-488. http://doi.org/10.5296/jsr.v3i2.2645

Bishop, R., Berryman, M., Wearmouth, J., Peter, M., \& Clapham, S. (2012). Professional development, changes in teacher practice and improvements in Indigenous students' educational performance: A case study from New Zealand. Teaching and Teacher Education, 28(5), 694-705. http://doi.org/10.1016/j.tate.2012.02.002

Blanco, C. \& Meneses, F. (2011). Estudiantes indígenas y educación superior en Chile: acceso y beneficios. In: EQUITAS (Ed.), Inclusión social, interculturalidad y equidad en la educación superior. Temuco.

Brunner, J. (2015). Medio Siglo de Transformaciones de la Educación Superior Chilena: Un estado del arte. In: Bernasconi, A. (Org.), La Educación Superior en Chile Transformación, Desarrollo y Crisis (p. 627). Santiago de Chile.

Carignan, N., Sanders, M., \& Pordavood, G. (2005). Racism and Ethnocentrism: Social Representations of Preservice Teachers in the Context of Multi-and Intercultural Education. International Journal of ..., 4(September), 1-17.

Chávez, M. E. (2008). Ser indígena en la educación superior ¿desventajas reales o asignadas?, XXXVII(148), 31-55.

Chirgwin, S. (2015). Burdens to difficult to carry? A case study of three academically able Indigenous Australian Masters students who had to withdraw. International Journal of Qualitative Studies in Education, 28(5), 594-609. http://doi.org/10.1080/09518398.20 14.916014

Cifuentes, R. (2015). Descentralización Político Administrativa y su Impacto en las Universidades Regionales Ricardo Cifuentes Lillo Subsecretario de Desarrollo Regional y Administrativo 2015. Revista Estudios Sociales sobre universidades regionales, 123.

Cook-Sather, A. (2015). Dialogue across differences of position, perspective, and identity: Reflective practice in/on a student-faculty pedagogical partnership program. Teachers College Record, 117(2). 
Cornejo, J. (2012). Educación, interculturalidad y ciudadanía Educacão, interculturalidade e cidadania Education, interculturality and citizenship. Educar em Revista, 43, 239-254.

Cupples, J. \& Glynn, K. (2014). Indigenizing and decolonizing higher education on Nicaragua's Atlantic Coast. Singapore Journal of Tropical Geography, 35, 56-71. http://doi.org/10.1111/sjtg.12051

Departamento de Extranjería y Migración Ministerio del interior y Seguridad Pública. (2016). Migración en Chile 2005 - 2014. Retrieved from www.extranjeria.gob.cl

Díaz, E. \& Careaga, R. (2010). Buenas prácticas del aseguramiento de la calidad de la Educación Superior en Chile. Santiago de Chile: Comisión Nacional de Educación - CNA.

Díaz-Aguado, M.J. (2004). Educación intercultural y cooperación. Una nueva interacción educativa para un mundo que también es diferente. Educatio Siglo XXI. Revista de la Facultad de Educación, (22), 59-89.

Die, G. (2011). Integrating Local Cultural Knowledge as Formal and Informal Education for Young African Learners: A Ghanaian Case Study. Canadian and International Education/Education,40(1), 20-40.

Diez, M.L. (2013). Repensando la interculturalidad en educación : aportes de la investigación socioantropológica a un campo problemático. Docencia, 51, 4-17.

Encuesta de caracterización Socioeconómica Nacional. 2013. Santiago de Chile: editorial MIDEPLAN.

Expósito, J., Olmedo, E., Pegalajar, M., \& Tomé, M. (2014). Pseudoconflicts in intercultural ESO (Mandatory Secondary Education ) classroom from the point of view of students and. Procedia - Social and Behavioral Sciences, 132, 100-106. http://doi.org/10.1016/j. sbspro.2014.04.284

Fernandes, L. \& Ferrão, M. (2013). Pedagogía Decolonial y Educación Anti-racista e Intercultural en Brasil. In: Walsh, C. (Org.), Pedagogías decoloniales: Prácticas insurgentes de resistir, (re) existir y (re) vivir. Tomo I (p. 507). Quito, Ecuador: Ediciones Abya-Yala.

Gómez, E.L. \& Navío, E.P. (2013). Formación Permanente Del Profesorado $Y$ Práctica Docente Intercultural: Contenidos Actitudinales $Y$ Complementariedad Competencial Service Teacher Training and Intercultural Teaching Practice: Attitudinal Contents and Integration of Competences. Espiral. Cuadernos del profesorado, 6(12), 32-42.

Hall, S.J. (2012). Deconstructing aspects of native speakerism: Reflections from in-service teacher education. The Journal of Asia Tefl, 9(3), 107-130.

Hall, M., Rata, A., \& Adds, P. (2013). He Manu Hou: The Transition of Māori Students into Māori Studies. International Indigenous Policy Journal, 4(4). http://doi.org/10.18584/iipj.2013.4.4.7
Hevia, R. (2010). El Derecho a la Educación y la Educación en Derechos Humanos en el contexto internacional. Revista Latinoamericana de Educación Inclusiva, 4, 25-39.

Kerr, J. (2014). Western epistemic dominance and colonial structures : Considerations for thought and practice in programs of teacher education. Descolonization: Indigeneity, Education \& Society, 3(2), 83-104.

Lea, J. (2000). The Macpherson Report and the Question of Institutional Racism. The Howard Journal, 39(3), 219-233. http:// doi.org/10.1111/1468-2311.00165

Lehtomäki, E., Moate, J., \& Posti-Ahokas, H. (2015). Global connectedness in higher education: student voices on the value of cross-cultural learning dialogue. Studies in Higher Education, 5079(December), 1-17. http://doi.org/10.1080/03075079.2015.1007943

Lewthwaite, B.E., Osborne, B., \& Boon, H. (2015). Seeking a Pedagogy of Difference: What Aboriginal Students and Their Parents in North Queensland Say About Teaching and Their Learning Seeking a Pedagogy of Difference: What Aboriginal Students and Their. Australian Journal of Teacher Education, 40(5). http://doi.org/10.14221/ajte.2015v40n5.8

López, J. (2012). Universidad e Inteculturalidad. In: Tubino, F.; Mansilla, K. (Orgs.), Universidad e Inteculturalidad, Desafios para América Latina. Lima: Pontificia Universidad Católca de Perú.

Mampaey, J. \& Zanoni, P. (2015). Reproducing monocultural education: ethnic majority staff's discursive constructions of monocultural school practices. British Journal of Sociology of Education, 1-19. http://doi.org/10.1080/01425692.2014.1001059

Meersohn, C. (2005). Introducción a Teun Van Dijk: Análisis de Discurso. Cinta de Moebio, 24, 288-302.

Mella, E.R., Rapiman, D.Q., Millan, S.Q., \& Antileo, E. (2016) Predominancia de la educación emocional occidental en contexto indígena: necesidad de una educación culturalmente pertinente. Psicologia Escolar e Educacional [online]. vol.20, n.3, pp. 523-532.

Merin, D.E. (2013). Reseña del libro El primer año universitario entre jóvenes provenientes de sectores de pobreza: un asunto de equidad, de Marisol Silva Laya y Adriana Rodríguez (2012), México, ANUIES (Biblioteca de la Educación Superior. Serie investigaciones). Revista Iberoamericana de Educación Superior (RIES), IV(10), 144-148.

Milne, T., Creedy, D.K., \& West, R. (2016). Integrated systematic review on educational strategies that promote academic success and resilience in undergraduate indigenous students. Nurse Education Today, 36, 387-394. http://doi.org/10.1016/j.nedt.2015.10.008

Ministerio de Educación Nacional (2009). Ley General de Educación. Chile. 
Navarrete, S., Candia, R., \& Puchi, R. (2013). Factores Asociados a La Deserción / Retención De Los Estudiantes Mapuche Académico. Calidad en la educación, 39(2), 44-80.

Niculescu, M. \& Percec, D. (2015). Intercultural Education in the Pre-service and In-service Teacher Training and Development. Procedia - Social and Behavioral Sciences, 180(0), 892-898. http://doi.org/http://dx.doi.org/10.1016/j.sbspro.2015.02.237

Ogbu, J.U. \& Simons, H.D. (1998). Voluntary and Involuntary Minorities: A Cultural-Ecological Theory of School Performance with Some Implications for Education. Anthropology \& Education Quarterly, 29(2), 155-188. http://doi.org/10.1525/aeq.1998.29.2.155

Oliver, R., Rochecouste, J., Bennell, D., Anderson, R., Cooper, I., Forrest, S., \& Exell, M. (2013). Understanding Australian Aboriginal tertiary student needs. International Jorunal of Higher Education, 2(4), 52-64. http://doi.org/http://dx.doi.org/10.5430/ijhe.v2n4p52

Olivera, I. (2013). ¿ Desarrollo o bien vivir ? Repensando la función social de la Universidad Intercultural desde el cuestionamiento al efecto educativo. Anthropologica, (33), 179-207.

Perrenoud, P. (2007). De la indiferencia ante las diferencias a las pedagogías diferenciadas. In: Perrenoud, P. Pedagogia diferenciada de las intenciones a la accion (214 p.). España: Editorial popular.

Quilaqueo, D. (2012). Saberes educativos mapuches: Racionalidad apoyada en la memoria social de los kimches. Atenea (Concepción) versión On-line, 505(1), 79-102. http://doi.org/10.4067/S071804622012000100004

Quilaqueo, D., Quintriqueo, S., \& Torres, H. (2014). Saberes Educativos Mapuches: Aportes. Chungará (Arica), 46(2), 271-284.

Quintriqueo, S. (2010). Implicancias de un modelo Curricular monocultural en contexto mapuche. Estudios pedagogicos. Temcuo: Universidad Católica de Temuco. http://doi.org/10.4067/ S0718-07052009000200010

Quintriqueo, S., Quilaqueo, D., Peña-, F., \& Muñoz, F. (2015).
Conocimientos Culturales como Contenido de la Educación Familiar Mapuche. Alpha, 40(2010), 131-146.

Schmelkes, S. (2010). Indígenas rurales, migrantes, urbanos: una educación equivocada, otra educación posible. Pensamiento Iberoamericano, 203-222.

Shoyer, S., Leshem, S., \& Boylan, M. (2016). Students' voice: The hopes and fears of student-teacher candidates. Cogent Education, 3(1), 1. http://doi.org/10.1080/2331186X.2016.1139438

Turra, O., Ferrada, D., \& Villena, A. (2013). La especificidad del contexto indígena como requerimiento para la formación inicial del profesorado. Estudios pedagógicos, XXXIX(2), 329-339.

Turra, O., Valdebenito, V., \& Torres, A. (2015). Teaching Competencies for Performing in school Vulnerability Contexts in Chile. Procedia Social and Behavioral Sciences, 197(February), 1236-1241. http:// doi.org/10.1016/j.sbspro.2015.07.386

Veldman, J., \& Guilfoyle, A. (2013). Racism and Resilience in Australian Aboriginal Graduates' Experiences of Higher Education. The International Journal of Learning in Higher Education, 19, 107-120.

Watson, L., Terrell, M.C., Wright, D.J., Bonner, F.A., Cuyjet, M.J., Gold, J., Rudy, \& Person, D. R. (2002). How Minority Students Experience College: Implications for Planning and Policy. (Stylus, Ed.). USA.

West, R., Usher, K., Foster, K., \& Stewart, L. (2014). Academic staff perceptions of factors underlying program completion by Australian Indigenous nursing students. The Qualitative Report, 19(12), 1-19.

Williamson, G. \& Navarrete, S. (2014). Cooperación internacional y educación intercultural bilingüe en Chile Cuatro casos en la educación superior. Revista Mexicana de Investigación Educativa, 19(60), 19-43.

Wit, H. de. (2011). Globalización e internacionalización de la educación superior. Revista de Universidad y Sociedad del Conocimiento, 8, 77-84.

Recebido em: 21 de novembro de 2016

Aprovado em: 08 de maio de 2017

\section{Sobre os autores}

Eliana Marcela Ortiz Velosa (eortiz@uct.cl)

Magister. Estudiante de Doctorado en Educación. Facultad de Educación. Universidad Católica de Temuco. Montt 56, Temuco, IX región, Chile.

Enrique Riquelme Mella (eriquelme@uct.cl)

Doctor. Universidad Católica de Temuco. Facultad de Educación. Montt 56, Temuco, IX región, Chile.

Se agradece el apoyo del proyecto FONDECYT 11140311, "socialización emocional en la educación familiar mapuche". 